\title{
Eye gaze is not unique: Automatic orienting in response to uninformative arrows
}

\author{
JASON TIPPLES \\ University of York, York, England
}

\begin{abstract}
Recent studies (Driver et al., 1999; Friesen \& Kingstone, 1998; Langton \& Bruce, 1999) have argued that the perception of eye gaze may be unique, as compared with other symbolic cues (e.g., arrows), in being able to automatically trigger attentional orienting. In Experiment 1, 17 participants took part in a visuospatial orienting task to investigate whether arrow cues might also trigger automatic orienting. Two arrow cues were presented for $75 \mathrm{msec}$ to the left and right of a fixation asterisk. After an interval of either 25 or $225 \mathrm{msec}$, the letter $\mathrm{O}$ or X appeared. After both time intervals, mean response times were reliably faster when the arrows pointed toward, rather than away from, the location of the target letter. This occurred despite the fact that the participants were informed that the arrows did not predict where the target would appear. In Experiment 2, the same pattern of data was recorded when several adjustments had been made in an attempt to rule out alternative explanations for the cuing effects. Overall, the findings suggest that the eye gaze is not unique in automatically triggering orienting.
\end{abstract}

A number of recent studies have shown that eye gaze can automatically trigger the orienting of attention (Driver et al., 1999; Friesen \& Kingstone, 1998; Langton \& Bruce, 1999). These findings are unique in the sense that other symbolic cues (e.g., arrows) do not appear to have such automatic effects (Jonides, 1981). Indeed, on the basis of these findings and neurophysiologicaldata (see Perrett \& Emery, 1994), it has been argued that automatic orienting to eye gaze may reflect the operation of a specialized cognitive mechanism. However, the present study reports automatic orienting to arrow cues and, therefore, demonstrates that eye gaze is not unique in automatically triggering attentional orienting.

In the visuospatial cuing task developed by Posner $(1978,1980)$, cues indicate the location of a target on valid trials and indicate a location away from the target on invalid trials. Under certain conditions, valid trials lead to reaction time benefits and invalid trials lead to reaction time costs. These cue validity effects are claimed to reflect the orienting of attention. On the basis of specific findings using the visuospatial cuing task, a distinction has been made between automatic orienting to exogenous cues and controlled orienting to endogenous cues.

A key feature of endogenous cues is that they are symbolic (e.g., $\rightarrow$ ) and therefore require interpretation. They are typically presented near the center of a display, and in

This research article was supported by a grant from the Medical Research Council. The author thanks Massimo Turatto, Tram Neill, and an anonymous reviewer for their helpful comments. Correspondence concerning this article should be sent to J. Tipples, Department of Psychology, University of Hull, Hull, HU6 7RX, England (e-mail: j.tipples@ hull.ac.uk). many studies, they correctly predict target locations on the majority of trials. Exogenous cues usually take the form of brief increases or decreases in luminance and appear directly in a possible target location. In comparison with endogenous cues, exogenous cues have a number of unique features that contribute to their automatic status. First, exogenous, but not endogenous, cues produce cuing effects despite having a low probability of correctly indicating target position and despite instructions that they be ignored (Jonides, 1981). Indeed, in a study by Remington, Johnston, and Yantis, (1992), exogenous cues produced shifts in visuospatial attention despite never appearing in the same location as the target. Furthermore, the effects of exogenous cues are reversed for cue-target intervals longer than $200 \mathrm{msec}$, producing short-lived inhibition at the cued location (inhibition of return; Posner \& Cohen, 1984). In contrast, the effects of endogenous cues take longer to emerge (approximately $300 \mathrm{msec}$; Müller \& Rabbitt, 1989). In summary, exogenous cues are difficult to ignore and rapidly instigate cuing effects, whereas endogenous cues can be ignored and are most effective in directing attention after a given period of time.

The effects of eye gaze information appear to be more similar to the automatic effects of exogenous cues than to the controlled effects associated with endogenous cues. Studies have found that eye gaze information (from a static image of a face), produces cue validity effects even when the direction of the eye gaze gives no information with respect to the location of the target stimulus (Driver et al., 1999; Friesen \& Kingstone, 1998; Langton \& Bruce, 1999). Furthermore, these studies have also shown that, like exogenous cues, eye gaze can trigger orienting at short cuetarget intervals. For example, Friesen and Kingstone pre- 
sented participants with a picture of a schematic face with the pupils removed from the eyes. After $680 \mathrm{msec}$, the pupils appeared within the eyes. The pupils were looking forward, to the left, or to the right. Following a further interval of $105,300,600$, or $1,005 \mathrm{msec}$, a target letter appeared to the left or to the right of the face. The target letters were equally likely to appear in the same or the opposite location to that indicated by the eye gaze. In separate tasks, participants were asked to detect, localize, and identify a target letter. In the 300-msec condition, participants were faster on valid trials than on invalid and neutral trials (eyes forward) for all three tasks. In the 105-msec condition, participants were faster on valid trials than on invalid and neutral trials for the detection and localization tasks. To reiterate, these findings suggest that eye gaze automatically triggers attentional orienting.

It is clear that the findings of Friesen and Kingstone (1998) and others are novel in the sense that single, uninformative, symbolic, centrally presented cues do not typically yield orienting effects. However, such a comparison may be unwarranted, on the grounds that eye gaze researchers present two cues (eyes) surrounding a fixation point (a nose; Friesen \& Kingstone, 1998), whereas researchers using arrow cues have typically presented a single arrow at fixation (e.g., Jonides, 1981). Arrow cues may trigger orienting more effectively when they are made more salient (by including two arrows rather than one arrow) and when they are not directly fixated. With respect to the last point, it may be more difficult to strategically override the effects of arrow cues if they are salient but not directly under the spotlight of attention.

\section{EXPERIMENT 1}

With the above points in mind, the first experiment reported here tested whether two spatially nonpredictive arrows can automatically trigger orienting in a manner similar to eye gaze information. Two arrows were presented either side of a fixation asterisk for a duration of $75 \mathrm{msec}$. The arrows were followed by an interval of either 25 or $225 \mathrm{msec}$ before the target appeared. Added to the cue duration, these intervals produced 100 - and 300-msec stimulus onset asynchrony (SOA) conditions. The two different SOA conditions were used to test the claim that orienting to symbolic cues is most effective at around $300 \mathrm{msec}$ before the onset of the target (Müller \& Rabbit, 1989). Target letters were equally likely to appear in the same or an opposite location to that indicated by the arrows. Following Friesen and Kingstone (1998), the participants were informed that the direction indicated by the arrows did not, in any way, predict the location of the response stimulus.

\section{Method}

Participants. Seventeen undergraduate psychology students (11 female, 6 male) from the University of York took part in exchange for course credit. All the participants had normal or corrected vision.

Apparatus and Stimuli. The outside arrows measured $1.1^{\circ}$ of visual angle in width and $0.9^{\circ}$ in height. These arrows were separated by a gap measuring $1.2^{\circ}$. The fixation asterisk $(*)$ was centered within this gap. The asterisk measured $0.7^{\circ}$ of visual angle in width and $0.7^{\circ}$ in height. Target letters were an uppercase $\mathrm{O}$ or $\mathrm{X}$, each subtending $0.9^{\circ}$ in height and width and appearing $5^{\circ}$ to either the left or the right of the center of the asterisk. The experiment was run on a Power Macintosh $(\mathrm{G} 3,233 \mathrm{~Hz})$ connected to a 19-in monitor. Psyscope (Cohen, MacWhinney, Flatt, \& Provost, 1993) was used to run the experiment. Each participant sat $60 \mathrm{~cm}$ away from the computer monitor in a darkened room. The experimenter ensured that the participants were positioned directly in front of the screen. The participants were also encouraged to keep their heads still throughout the experiment.

Design. The participants undertook 10 blocks of 16 trials. The first block was treated as practice and was excluded from the data analysis. Each block consisted of equiprobable factorial combinations of SOA (100 or $300 \mathrm{msec}$ ), cue direction (left or right), target position (left or right) and target type (O or X). Within each block, the trials were presented in a different random order for each participant. After the practice trials, the participants were allowed a brief rest before starting the main block of trials

Procedure. Prior to starting the experiment and during the rest period, certain aspects of the instructions were emphasized. First, the participants were told to fix their eyes on a central asterisk. Second, speedy and accurate responding was encouraged. Finally, the participants were told that the direction indicated by the arrows did not, in any way, predict the location of the response stimulus.

An example of the trial sequence is displayed in Figure 1. Each trial began with a central fixation asterisk. The central fixation asterisk remained present throughout each trial. After $675 \mathrm{msec}$, the arrows appeared for a duration of $75 \mathrm{msec}$. Following the arrows, there was an interval of either 25 or $225 \mathrm{msec}$, during which the asterisk remained on the screen. Following the interval, a target letter appeared to either the left or the right of the asterisk. The target remained present until a response was made. With respect to the target response, the participants made a forced-choice target discrimination by pressing the $\mathrm{H}$ key or the spacebar for the $\mathrm{X}$ and $\mathrm{O}$ targets, respectively ( $\mathrm{O}$ and $\mathrm{X}$ labels were placed on the appropriate keys). Responses were made with the dominant hand, using the index finger (for X targets) and the thumb (for $\mathrm{O}$ targets). Feedback for correct $(+)$ and incorrect $(-)$ responses were presented after the target response. The feedback appeared in the same location as the asterisk, thus encouraging the participants to stay fixated in the center throughout each trial. The feedback display was presented for $675 \mathrm{msec}$ before the fixation asterisk indicated that a new trial had begun.

\section{Results}

Incorrect responses (5\%) were excluded from reaction time analysis. In addition, for each participant, reaction times more than three standard deviations above or below the mean in each of the experimental conditions were treated as outliers and were excluded from the data analyses. This procedure resulted in the exclusion of $1.2 \%$ of the trials. The remaining mean correct reaction times were analyzed in a cue validity (valid or invalid) $\times$ SOA $(100$ or $300 \mathrm{msec})$ repeated measures analysis of variance (ANOVA). The mean correct reaction times and mean proportion of errors as a function of cue validity and SOA are displayed in Table 1 . The reaction time analyses revealed main effects for cue validity $\left[F(1,16)=23.73, M S_{\mathrm{e}}=188, p<.0005\right.$, and SOA $\left[F(1,16)=121.98, M S_{\mathrm{e}}=389, p<.0001\right]$. Cue validity did not interact with SOA $\left[F(1,16)=0.06, M S_{\mathrm{e}}=\right.$ $379, p>.5]$.

The main effect of cue validity reflected faster reaction times on valid trials $(M=469 \mathrm{msec})$ than on invalid trials $(M=485 \mathrm{msec})$. The main effect of SOA reflected faster 


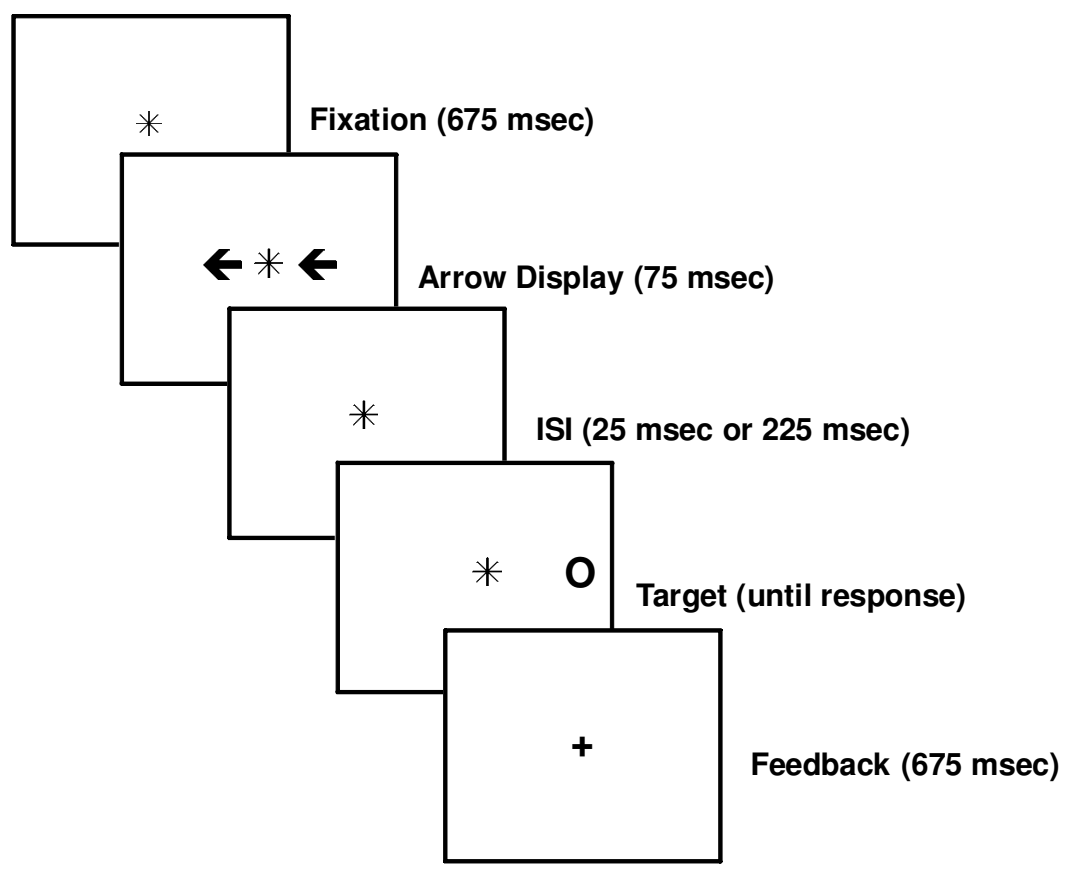

Figure 1. Experiment 1: a typical (invalid) trial sequence with successive events running from top left to bottom right. See the Procedure section for more details. Stimuli are not depicted to scale.

reaction times in the 300-msec SOA condition $(M=$ $451 \mathrm{msec})$ than in the $100-\mathrm{msec}$ SOA condition $(M=$ $503 \mathrm{msec}$ ). The latter effect was assumed to reflect a nonspatial, temporal warning effect (Posner, 1978). Planned contrasts were conducted to examine the effect of cue validity for each of the SOA conditions. In line with the hypothesis that arrows automatically trigger orienting, the main effect of cue validity was significant in both the 100 msec SOA condition $\left[F(1,16)=7.01, M S_{\mathrm{e}}=364, p<\right.$ $.05]$ and the 300-msec SOA condition $[F(1,16)=9.51$, $\left.M S_{\mathrm{e}}=282, p<.01\right]$. The mean proportion of errors did not vary systematically with either cue validity or SOA (largest $F=1.87$, n.s.). Importantly, error rates were not higher in either of the valid cuing conditions, suggesting that a speed-accuracy tradeoff cannot account for the observed pattern of data.

Table 1

Mean Correct Reaction Time (RT) and Mean Proportion of Error (PE) for Experiment 1 as a Function of Cue Validity and Stimulus Onset Asynchrony (100- or 300-msec SOA)

\begin{tabular}{ccccc}
\hline \multirow{2}{*}{ SOA $(\mathrm{msec})$} & \multicolumn{2}{c}{$\mathrm{RT}$} & \multicolumn{2}{c}{ PE } \\
\cline { 2 - 5 } \cline { 2 - 5 } & $M$ & $S D$ & $M$ & $S D$ \\
\hline \multirow{2}{*}{100} & 495 & 56 & .06 & .06 \\
300 & 443 & 68 & .04 & .04 \\
& \multicolumn{4}{c}{ Valid Cues } \\
100 & 513 & 68 & .06 & .06 \\
300 & 459 & 67 & .06 & .03 \\
\hline
\end{tabular}

\section{Discussion}

In Experiment 1, the participants responded more quickly following valid arrow cues, as compared with invalid arrow cues. This effect was found in both the 100- and the $300-\mathrm{msec}$ SOA conditions. Clearly, then, uninformative arrow cues automatically trigger the orienting of attention. Therefore, orienting to eye gaze at short cue-target intervals does not necessarily reflect the operation of a specialized mechanism that has different functional properties to other types of uninformative symbolic cues.

\section{EXPERIMENT 2}

Experiment 2 addressed several alternative reasons for the cuing effects recorded in Experiment 1. One possible explanation for the cuing effects relates to a specific perceptual characteristic of the arrow stimuli. The arrows contain more perceptual information around the arrowhead than around the tail of the arrow. Therefore, on a valid trial, for example, the greater weight of perceptual information nearer the target may have stimulated attention to a greater extent in the cued location than in the uncued location. To correct for this imbalance, the tail was trimmed off the arrow to create a more symmetrical arrow. A further possible problem with the stimuli used in Experiment 1 relates to the overall configuration of the arrows and asterisk. It might be argued that the asterisk in combination with the arrows creates a configuration that resembles the nose and eyes on a face (with the asterisk serving as the nose and the arrows serving as the eyes). 
Table 2

Mean Correct Reaction Time (RT) and Mean Proportion of Error (PE) for Experiment 2 as a Function of Cue Validity and Stimulus Onset Asynchrony (100- or 300-msec SOA)

\begin{tabular}{lllll}
\hline \multirow{2}{*}{ SOA } & \multicolumn{2}{c}{ RT } & \multicolumn{2}{c}{ PE } \\
\cline { 2 - 5 } \cline { 3 - 5 } & $M$ & \multicolumn{5}{c}{ Valid Cues } \\
\hline \multirow{5}{*}{100} & 524 & 78 & .05 & .06 \\
300 & 489 & 68 & .05 & .06 \\
& \multicolumn{5}{c}{ Invalid Cues } \\
100 & 547 & 90 & .05 & .04 \\
300 & 503 & 71 & .06 & .06 \\
\hline
\end{tabular}

Experiment 2 makes the stimulus display less facelike by placing the fixation asterisk above the arrows. Noses do not appear above the eyes in a face, and therefore, such a configuration is unlikely to trigger processes specific to eye gaze. One further change followed the procedure used by Driver et al. (1999) and Friesen and Kingstone (1998). Specifically, the arrows remained on the screen until the participant made a response. As was noted by Driver et al., the brief presentation of a spatial cue creates a salient visual transient that may reduce or eliminate the desired cuing effects. Leaving the arrows on the screen should lessen any attentional costs associated with the sudden offset of the arrows.

\section{Method}

Participants. Twenty-one undergraduate psychology students (13 female, 8 male) from the University of York took part in exchange for course credit. All the participants had normal or corrected vision.

Stimuli, Design, and Procedure. Three adjustments were made in Experiment 2. First, to prevent the asterisk and arrows from resembling the nose and eyes of a face, the fixation asterisk and feedback signals $\left(+\right.$ or - ) were both raised $0.5^{\circ}$ above the position used in Experiment 1. Second, to create a more symmetrical arrow, the tail of the arrow was trimmed so that the total length of the arrow was now equal to the length of the arrowhead. Finally, the arrows remained on display until the participant responded. Apart from these differences, Experiment 2 was identical to Experiment 1.

\section{Results}

Incorrect responses $(5 \%)$ were excluded from the reaction time analyses. Outliers (2\%) were excluded, using the criterion adopted in Experiment 1. The remaining mean correct reaction times were analyzed in a cue validity (valid or invalid) $\times$ SOA $(100$ or $300 \mathrm{msec})$ repeated measures ANOVA. The mean correct reaction times and mean proportion of errors as a function of cue validity and SOA are displayed in Table 2. As in Experiment 1, the reaction time analyses revealed main effects for cue validity $\left[F(1,20)=14.48, M S_{\mathrm{e}}=482, p<.005\right]$ and SOA $\left[F(1,20)=41.55, M S_{\mathrm{e}}=773, p<.0001\right]$. Cue validity did not interact with SOA $\left[F(1,20)<0.5, M S_{\mathrm{e}}=843, p>.5\right]$.

The main effect of cue validity reflected faster reaction times on valid trials $(M=506 \mathrm{msec})$ than on invalid trials $(M=525 \mathrm{msec})$. The main effect of SOA reflected faster reaction times in the $300-\mathrm{msec}$ SOA condition $(M=$
$496 \mathrm{msec})$ than in the 100-msec SOA condition $(M=$ $535 \mathrm{msec}$ ). As in Experiment 1, planned contrasts were conducted to examine the effect of cue validity for each of the SOA conditions. The main effect of cue validity was significant in the 300-msec SOA condition $[F(1,20)=$ $\left.4.53, M S_{\mathrm{e}}=467, p<.05\right]$ and the $100-\mathrm{msec}$ SOA condition $\left[F(1,20)=6.08, M S_{\mathrm{e}}=858, p<.05\right]$. The mean proportion of errors did not vary systematically with either cue validity or SOA (largest $F=.85$, n.s.). Again, there was no evidence of a speed-accuracy tradeoff.

\section{Discussion}

The data from Experiment 2 provide further support for the hypothesis that arrow stimuli can automatically trigger orienting. The participants were faster to react on valid than on invalid trials irrespective of whether the arrows appeared 100 or $300 \mathrm{msec}$ before the target letter. These effects occurred under new conditions in which the arrows no longer contained more perceptual information around the arrowhead and in which adjustments were made to make the display look less facelike. In summary, automatic orienting to arrows appears to be a replicable finding.

\section{GENERAL DISCUSSION}

The findings from two experiments show that two arrows can automatically trigger the orienting of attention. In Experiment 1 , the participants were faster to detect targets in briefly cued locations than in uncued locations. This effect was found in the 100- and 300-msec SOA conditions. The fact that the orienting effects occurred after only $100 \mathrm{msec}$ and that the participants were explicitly informed that the cues were uninformative suggests that orienting in response to arrow cues is an automatic phenomenon. This effect was replicated in a further experiment in which an attempt was made to control unwanted perceptual characteristics of the arrows. Moreover, the facelike qualities of the display were changed so that the display could no longer be mistaken for facial features. These findings reinforce the conclusion that eye gaze is not unique in automatically triggering orienting. Furthermore, they contradict earlier research that showed that uninformative symbolic cues do not automatically trigger orienting (Jonides, 1981).

Given that arrows are used in situations in which humans need to rapidly direct attention (e.g., in reaction to road signs), it is somewhat surprising that earlier research failed to find automatic orienting to arrow cues. Indeed, there may be several reasons why orienting effects were recorded in the present experiments. For example, the arrows in the present experiments were not directly under the spotlight of visual attention. In earlier research (e.g., Jonides, 1981), the arrow appeared in the same location as a fixation dot. Such a procedure may affect the holistic processing of the arrow by concentrating visual attention on a specific part of the arrow (e.g., the tail), rather on than the whole arrow. In the present experiments, the arrows were presented slightly away from fixation, in a 
manner that may have enabled the holistic processing of the arrows. In a similar vein, the effects of arrow cues placed away from fixation may have influenced responding because they were less susceptible to conscious processing strategies. An alternative explanation relates to the fact that the present experiments used two arrows, rather than the one arrow used in earlier research (e.g., Jonides, 1981). Put simply, two arrows may have greater informational value than does one arrow and, consequently, have stronger effects on orienting. In summary, presenting the arrows slightly away from fixation and presenting two arrows rather than one arrow are notable differences between the present study and earlier research. Either of these differences may have contributed to the effects reported here and are worth examining in future research.

In summary, the present study reports automatic orienting in response to arrow cues. These findings should not be taken as evidence against the importance of eye gaze. The perception of eye gaze direction may play a role in the formation of a theory of mind (Baron-Cohen, 1995) and participation in episodes of joint attention (Bruner, 1983). Furthermore, it seems reasonable to argue that such abilities may have produced an adaptive advantage across human evolutionary history. However, further experimentation is needed before it can be claimed that eye gaze triggers orienting in a unique fashion, as compared with other symbolic cues.

\section{REFERENCES}

Baron-Cohen, S. (1995). The eye direction detector (EDD) and the shared attention mechanism (SAM): Two cases for evolutionary psychology. In C. Moore \& P. Dunham (Eds.), Joint attention: Its origins and role in development (pp. 41-59). Hillsdale, NJ: Erlbaum.
BRUner, J. (1983). Child's talk: Learning to use language. Oxford: Oxford University Press.

Cohen, J. D., MacWhinney, B., Flatt, M., \& Provost, J. (1993). PsyScope: An interactive graphic for designing and controlling experiments in the psychology laboratory using Macintosh computers. Behavioral Research Methods, Instruments, \& Computers, 25, 257271.

Driver, J., Davis, G., Ricciardelli, P., Kidd, P., Maxwell, E., \& BARon-Cohen, S. (1999). Gaze perception triggers reflexive visuospatial orienting. Visual Cognition, 6, 509-540.

Friesen, C. K., \& Kingstone, A. (1998). The eyes have it! Reflexive orienting is triggered by nonpredictive gaze. Psychonomic Bulletin \& Review, 5, 490-495.

JoNIDES, J. (1981). Voluntary versus automatic control over the mind's eye's movement. In J. [B.] Long \& A. [D.] Baddeley (Eds.), Attention and performance IX (pp. 187-203). Hillsdale, NJ: Erlbaum.

LANGTON, S. R. H., \& BRUCE, V. (1999). Reflexive visual orienting in response to the social attention of others. Visual Cognition, 6, 541-567.

Müller, H. J., \& Rabbitt, P. M. A. (1989). Reflexive and voluntary orienting of visual attention: Time course of activation and resistance to interruption. Journal of Experimental Psychology: Human Perception \& Performance, 15, 315-330.

Perrett, D., \& Emery, N. J. (1994). Understanding the intentions of others from visual signals: Neuropsychological evidence. Cahiers de Psychologie Cognitive, 13, 683-694.

Posner, M. I. (1978). Chronometric explorations of mind. Hillsdale, NJ: Erlbaum.

Posner, M. I. (1980). Orienting of attention. Quarterly Journal of Experimental Psychology, 32A, 3-25.

Posner, M. I., \& Cohen, Y. (1984). Components of visual orienting. In H. Bouma \& D. G. Bowhuis (Eds.), Attention andperformance X: Control of language processes (pp. 531-556). Hillsdale, NJ: Erlbaum.

Remington, R. W., Johnston, J. C., \& Yantis, S. (1992). Involuntary attentional capture by abrupt onsets. Perception \& Psychophysics, 51, 279-290.

(Manuscript received November 9, 2000; revision accepted for publication June 26, 2001.) 